\title{
BIOCHEMICAL MARKERS OF THE MECHANICAL QUALITY OF ENGINEERED HYALINE CARTILAGE
}

D.J. Kelly ${ }^{1}$, A. Crawford ${ }^{2}$, S. C. Dickinson ${ }^{3}$, T. J. Sims ${ }^{3}$, J. Mundy ${ }^{2}$, A. P. Hollander $^{3}$, P.J.Prendergast ${ }^{1}$ and P.V. Hatton ${ }^{2}$.

${ }^{1}$ Dept of Bioengineering, Trinity College, Dublin, Ireland.

${ }^{2}$ Centre for Biomaterials and Tissue Engineering, University of Sheffield, Sheffield. UK.

${ }^{3}$ Academic Rheumatology, University of Bristol, Avon Orthopaedic Centre, Southmead Hospital, Bristol. UK. 


\begin{abstract}
The aim of this study was to determine whether or not biochemical markers can be used as surrogate measures for the mechanical quality of tissue engineered cartilage. The biochemical composition of tissue engineered cartilage constructs were altered by varying either (i) the initial cell seeding density of the scaffold (seeding density protocol) or (ii) the length of time the engineered tissue was cultured (culture period protocol). The aggregate or Young's moduli of the constructs were measured (by confined or unconfined compression respectively), and compared with the composition of the extracellular matrix by quantitative measurement of the glycosaminoglycan (GAG), hydroxyproline, collagen I and collagen II and collagen cross-links. The aggregate modulus correlated positively with both GAG and collagen II content, but not with collagen I content. Young's modulus correlated positively with GAG, collagen II and collagen I content, and the ratio of mature to immature cross-links, but not with hydroxyproline content. These results suggested that hydroxyproline may be an unreliable indicator of mechanical quality of tissue engineered cartilage, and that a measure of collagen II and GAG content is required to predict the biomechanical quality of tissue engineered cartilage.
\end{abstract}




\section{Introduction}

The mechanical behaviour of cartilage is closely related to its biochemical content [1]. In normal articular cartilage, structural macromolecules (primarily type II collagen and proteoglycan) interact to form a porous, saturated, fibre-reinforced matrix capable of withstanding the mechanical forces associated with joint articulation. The tensile strength of the tissue is attributed primarily to the type II collagen, aided by crosslinking of the collagen fibrils, while proteoglycans contribute primarily to the compressive properties of the tissue by two means: a swelling pressure associated with the charged nature of proteoclycan aggregates [2], and the bulk compressive stiffness of the proteogylcan aggregates immobilised within the collagen network. The collagen-proteoglycan matrix also has a very low permeability to fluid flow, and it has been shown that during loading over $90 \%$ of the load is initially supported by the build-up of interstitial fluid pressure [3]. Similar structure-function relationships have been shown to exist for tissue engineered cartilage; however it is still unclear what biochemical markers are the most appropriate indicators of the mechanical properties of the tissue.

A number of studies have reported changes in the composition of cartilage constructs engineered in vitro, typically assessed by glycosaminoglycan (GAG) content and/or total collagen determined from hydroxyproline content, with associated changes in the tissues mechanical properties [4-19]. For example, Hunter et al. [4] correlated the dynamic stiffness of engineered cartilage with both GAG and hydroxyproline content. In their study the GAG content correlated positively with the hydroxyproline content, and the DNA content correlated positively with both GAG and hydroxyproline contents, making it difficult to associate changes in dynamic stiffness with specific changes in construct composition rather than general maturation effects. Mauck et al. [5] observed that the aggregate modulus and Young's 
modulus of tissue engineered cartilage constructs depend more strongly on collagen content than GAG content. Vunjak-Novakovic et al. [6] were able to use multiple linear regression analysis to correlate changes in the mechanical properties (equilibrium modulus, hydraulic permeability and dynamic stiffness) of tissue engineered cartilage to changes in the amounts of glycosaminoglycan, collagen and water. However the differences between the mechanical properties of the engineered tissues and freshly explanted tissue could not be solely explained by differences in the amounts of collagen and glycosaminolycan, implying to the authors that either the accumulation of glycosaminoglycan and collagen precede their assembly into a functional tissue, or that the assembly of the extracellular matrix was different from that in natural cartilage. It would therefore seem that assessing engineered cartilage by GAG and hydroxyproline content alone may not be a sufficient indicator of its biomechanical quality.

Type II collagen accounts for more than $90 \%$ of the total collagen in hyaline cartilage whereas type I collagen is abundant in fibrocartilage but largely absent from normal, mature hyaline cartilage. However both collagen types are usually present in tissue engineered cartilage. Dickinson et al. [20] have developed quantitative inhibition ELISA assays for both type I and II collagens which allow the measurement of both collagen types following the enzymic digestion of tissue engineered cartilage. Total collagen, as hydroxyproline, mature and immature collagen cross-links and GAG can also be measured in the same digests, thereby allowing the mechanical properties of tissue engineered cartilage to be correlated with individual matrix components.

The aim of this study was to determine whether or not biochemical markers can be used as surrogate measures for the mechanical quality of tissue engineered 
cartilage. To this end, two separate experimental protocols were designed with the specific objective of engineering cartilage constructs with differing extracellular matrix composition, and hence differing mechanical properties. By varying either the initial cell seeding density or the in vitro culture period, it was possible to alter the biochemical composition of tissue engineered cartilage constructs, and to then correlate changes in GAG, collagen I, collagen II, and collagen cross-linking with the mechanical properties of the construct. If such a correlation exists then it would indicate the most important biochemical markers to test for in tissue engineered cartilage.

\section{Methods}

\section{Experimental design}

This study consisted of two separate study protocols: (i) a cell seeding density protocol, in which the initial density of cells used to seed a scaffold was varied and (ii) varying the maturity of the engineered constructs by varying the length of time constructs were cultured, (in this condition, the scaffolds were seeded with the same cell density). For the first study protocol, bovine articular chondrocytes were seeded onto scaffolds ( $2 \mathrm{~mm}$ depth, $5 \mathrm{~mm}$ diameter) with four cell seeding densities of 2, 4, 8 and 16 million cells/scaffold. After 42 days of culture, the constructs were tested in confined compression prior to biochemical analysis to determine GAG, collagen I and collagen II content. Five constructs were obtained for each seeding density by repeating the experiment on separate occasions using cells isolated from different animals. In the second study protocol (i.e. varying the length of time in culture), bovine nasal chondrocytes were seeded onto scaffolds ( $2 \mathrm{~mm}$ depth, $8 \mathrm{~mm}$ diameter) at a seeding density of 15 million cells/scaffold, and then cultured for up to 80 days. 
Constructs were tested in unconfined compression after 20,30, 40 and 80 days in culture, followed by biochemical analysis to determine GAG, collagen I, collagen II, hydroxyproline content and the ratio of immature to mature collagen cross-links. This study protocol was performed in two laboratories (Universities of Sheffield and Bristol) (termed study A and B in the text) using cells from different animals and using different batches of sera to obtain an average of 15 constructs per time point. Again to obtain this number of samples per time point required repeating the experiment on separate occasions using cells isolated from the tissue of different animals.

The mechanical properties of the engineered tissues where determined using either confined compression to quantify the aggregate modulus, or unconfined compression to quantify the Young's modulus (see Biomechanical evaluation section below). In confined compression testing, the tissue is loaded purely in compression, whereas in unconfined compression the sample is allowed to bulge radially and tensile strains are generated in the tissue. Since proteoglycans are commonly considered to resist compression, and collagen to resist tension, it was deemed necessary to examine correlations between both the Young's modulus and the aggregate modulus with biochemical markers for GAG, collagen I, collagen II, and collagen cross-linking.

\section{Isolation and culture of bovine chondrocytes}

Full thickness hyaline cartilage was harvested from bovine metacarpophalangeal joints or nasal cartilage from adult animals (18-24 months) within 4 hours of slaughter. Chondrocytes were isolated from all cartilages as described previously [21]. In brief, chondrocytes were released by sequential proteolytic digestion at $37^{\circ} \mathrm{C}$ for 30 min. in trypsin $(0.25 \%$ in PBS, Invitrogen Ltd. UK) followed by incubation for 
18-22 h with $0.2 \%$ bacterial collagenase (Sigma, UK) in basic culture medium (Dulbecco's modified Eagles medium (DMEM, Invitrogen, UK), containing $10 \mathrm{mM}$ HEPES (Invitrogen, UK) 10\% FBS, non essential amino acids, 100 units/ml penicillin and $100 \mu \mathrm{g} / \mathrm{ml}$ streptomycin sulphate (Sigma, UK). The isolated cells were seeded in tissue culture plates $\left(3 \times 10^{4}\right.$ cells $\left./ \mathrm{cm}^{2}\right)$ and cell numbers expanded for two to three passages in expansion medium (basic medium containing $10 \mathrm{ng} / \mathrm{ml}$ FGF-2, PreproTech, UK).

\section{Formation and culture of engineered constructs}

Chondrocyte/scaffold constructs were engineered and cultured as described previously [21]. Briefly, scaffolds of a non-woven fleece $(8 \mathrm{~mm}$ diameter, $2 \mathrm{~mm}$ thick) of Hyaff $11^{\circledR}$, Fidia Advanced Biopolymers, Italy); were threaded onto stainless steel wires and suspended in a flask containing a gently stirred suspension (70 rpm) of chondrocytes in expansion medium for $72 \mathrm{~h}$. Scaffolds were seeded with the initial chondrocyte concentration stated above. Freshly seeded constructs were transferred to petri-dishes coated with $1 \%$ agarose (Sigma, to prevent cell adhesion) and incubated in expansion medium for a further 4 days before being transferred into differentiation medium [basic medium supplemented with $10 \mu \mathrm{g} / \mathrm{ml}$ insulin (Sigma) and $50 \mu \mathrm{g} / \mathrm{ml}$ ascorbic acid (Sigma)] for the remainder of the culture period. Media was changed every 3-4 days and the culture dishes were placed on an orbital shaker (50 rpm) for the duration of incubation. For the cell seeding protocol, the constructs were incubated for 42 days in studies carried out in one laboratory (University of Sheffield). For the time-in-culture protocol, the constructs were incubated for 20, 30, 40 or 80 days following cell seeding in studies carried out in two laboratories (Sheffield and Bristol Universities). Constructs were transported (within 24h at room 
temperature) to Trinity College Dublin in basic culture medium for biomechanical analysis. After mechanical testing, constructs were stored at $-20^{\circ} \mathrm{C}$ while awaiting biochemical analyses (University of Bristol).

\section{Biomechanical evaluation}

Discs (3 mm diameter) were cored from the central region of engineered constructs from the cell seeding protocol, and placed in a cylindrical confining chamber. The chamber was mounted onto a Zwick Z005 materials testing machine, and the sample was compressed by a porous sintered bronze platen (Aegis Advanced Materials, Worcestershire, UK). Samples were immersed in normal saline solution at room temperature throughout the test. A ramp displacement corresponding to $5 \%$ strain at a ramp speed of $0.001 \mathrm{~mm} / \mathrm{sec}$ was applied to each sample, which was then held until the measured reaction force equilibrated (Fig. 1a). Two subsequent ramp displacements of 5\% strain each were then applied to each sample, giving a total strain of $15 \%$. Based on the magnitude of the equilibrium force measured after each ramp and hold, a stress-strain curve for the tissue can be obtained. The equilibrium modulus in confined compression (termed the aggregate modulus) of the tissue is obtained from the slope of the stress-strain curve (Fig. 1b), which can be considered linear for strains below $15 \%$.

At each harvest point (20, 30, 40 and 80 days), samples from the time-inculture experiment were tested in unconfined compression between two polished stainless steel platens mounted onto a Zwick Z005 materials testing machine. Samples were immersed in normal saline solution at room temperature throughout the test. The

same loading regime as used for the confined compression test was used for the unconfined compression test. For unconfined compression testing, the whole 
construct was tested, as apposed to confined compression testing where a $3 \mathrm{~mm}$ disc was cored out (Note: Biochemical assays were only performed on the cored discs and not the total construct). In this case, the equilibrium modulus in unconfined compression (termed the Young's modulus) of the tissue is obtained from the slope of the stress-strain curve.

\section{Biochemical analysis}

Trypsin Digestion - Following biomechanical evaluation, constructs obtained from both the cell seeding density and time-in-culture experiments were freeze-dried and weighed to obtain the dry weight. All samples were then digested overnight at $37^{\circ} \mathrm{C}$ with $250 \mu \mathrm{l}$ of TPCK-treated bovine pancreatic trypsin prepared at $2 \mathrm{mg} / \mathrm{ml}$ in Tris buffer, $\mathrm{pH} 7.5$, containing $1 \mathrm{mM}$ iodoacetamide, $1 \mathrm{mM}$ EDTA and $10 \mu \mathrm{M}$ pepstatin $\mathrm{A}$ (all from Sigma). A further $250 \mu \mathrm{l}$ of freshly prepared trypsin was added to each sample and there was a further incubation for $2 \mathrm{~h}$ at $65^{\circ} \mathrm{C}$ [20]. All samples were boiled for $15 \mathrm{~min}$ at the end of incubation, to destroy any remaining enzyme activity. Undigested material was removed, freeze-dried and weighed. The supernatants were assayed for matrix components as described below.

Type I collagen - The digests were assayed by inhibition ELISA using a rabbit antipeptide antibody to type I collagen, as previously described [20]. Peptide SFLPQPPQ was synthesised by Dr. A. Moir (Kreb's Institute, Sheffield University, UK) and was used as a standard in all of the immunoassays.

Type II collagen - The digests were assayed by inhibition ELISA using a mouse IgG monoclonal antibody to denatured type II collagen, COL2-3/4m, as previously 
described [20,22]. Peptide CB11B (CGKVGPSGAP[OH]GEDGRP[OH]GPP[OH] GPQY) was synthesised by Dr. A. Moir (Kreb's Institute, Sheffield University, UK) and was used as a standard in all of the immunoassays.

Total collagen - Total collagen in the digests was measured as hydroxyproline by amino acid analysis [23].

Collagen cross-links - The immature hydroxylysino-5-ketonorleucine and the mature hydroxylysyl-pyridinoline cross-links were measured by amino acid analysis, as previously described [20, 23]. Briefly, samples were reduced with sodium borohydride to stabilise collagen cross-links to heat and acid and then hydrolysed in constant boiling hydrochloric acid. The hydrolysates were applied to CF1 cellulose chromatography columns to remove non-cross-linking amino acids and to concentrate the collagen cross-links. These were then separated and quantified using a Biochrom20 Plus amino acid analyser equipped with ninhydrin detection and configured for the separation of collagen cross-links.

Proteoglycan - A colorimetric assay for GAG, using dimethylmethylene blue, was used to measure proteoglycans in the digests [24].

\section{Statistical analysis}

Mechanical and biochemical properties of engineered constructs are expressed as the average \pm SEM. Differences in mechanical and biochemical properties with seeding density or time-in-culture were determined by one-factor ANOVA with post-hoc Tukey test. Differences in mechanical and biochemical properties between the timein-culture studies by the two laboratories were determined by two-factor ANOVA 
with Bonferroni post tests. Correlations between mechanical properties (Aggregate modulus or Young's modulus) and biochemical content (collagen I, collagen II, GAG, collagen cross-linking and hydroxyproline) were determined using a Spearman correlation (significant at or below $P=0.05$ ). The Spearman correlation coefficient $r$ is expressed as a $95 \%$ confidence interval.

\section{Results}

Confined compression did not reveal any statistical difference between the mean aggregate modulus of constructs seeded at different cell densities (Fig. 2a). No statistical difference or trends were observed between the collagen I levels of these constructs (Fig. 2b). For collagen II and GAG, a general trend was observed where the collagen II and GAG levels (expressed as a percentage of construct matrix dry weight) increased using cell seeding densities of 4 and 8 million cells (Fig. 2c, 2d). However, this trend did not reach statistical significance for collagen II although the GAG content at a seeding density of 4 million cells/scaffold was significantly higher than at 16 million cells/scaffold $(\mathrm{P}<0.05)$ (Fig. 2d).

For the cell seeding studies, the aggregate modulus correlated positively with both GAG $(P<0.0001, r=0.5737$ to 0.9147$)$ and Collagen II $(P<0.0001, r=0.6215$ to 0.9261 ) content (all expressed as a percentage of construct dry weight), but not with Collagen I content (Fig. 3).

For the time-in-culture studies, the cell seeding density was kept constant and the period of time-in-culture varied. The period of time the constructs were cultured had an effect $(\mathrm{P}<0.05)$ on the Young's modulus. For study A, Young's modulus after 40 days in culture was greater than after $20(\mathrm{P}<0.001)$ or $30(\mathrm{P}<0.01)$ days in culture, but there was no significant difference $(P>0.05)$ between the Young's 
modulus at day 40 and day 80 (Fig. 4a). For study B, Young's modulus after 80 days was greater than the modulus after 30 days $(\mathrm{P}<0.01)$. The Young's modulus of the constructs from study A was greater $(\mathrm{P}<0.001)$ than the Young's modulus from study B at all time points except at day 20 . The length of time that the constructs were cultured also had a significant effect $(\mathrm{P}<0.05)$ on the collagen I (Fig. 4b), collagen II (Fig. 4c), GAG (Fig. 4d), and hydroxyproline (Fig. 4f) content of the matrix (all expressed as a percentage of the construct matrix dry weight), and the ratio of mature to immature cross-links in both the original and replicate experiment (Fig. 4e), except in study B where no difference in GAG content as a percentage of construct matrix dry weight was observed with time in culture. There was no difference in biochemical content, expressed as percentage of construct dry weight, between the original and replicate experiment, except at day 80 when there was a difference in GAG content $(\mathrm{P}<0.05)$ and the ratio of mature to immature cross-links $(\mathrm{P}<0.001)$.

For the culture period experiment, the Young's modulus correlated positively with GAG $(P<0.0001, r=0.3926$ to 0.7375$)$, collagen II $(P<0.0001, r=0.4268$ to 0.7557) and collagen I ( $P=0.003, r=0.2188$ to 0.6366$)$ content (all expressed as a percentage of construct dry weight), and the ratio of mature to immature cross-links ( $P<0.0001, r=0.2802$ to 0.6739$)$, but not with hydroxyproline content (Fig. 5).

\section{Discussion}

The principal objective of this study was to determine the most appropriate biochemical markers for the mechanical quality of tissue engineered cartilage. The mechanical properties of tissue engineered cartilage were correlated with biochemical content, which were expressed as a percentage of total construct dry weight. By 
identifying such correlations, it should be possible to better predict the mechanical quality of tissue engineered cartilage from small biopsies.

In this study, cartilage tissue was engineered from both bovine articular chondrocytes and bovine nasal chondrocytes. A number of studies have demonstrated the potential of nasal chondrocytes for cartilage tissue engineering [25, 26, 27]. Nasal chondrocytes have been shown to generate a matrix with significantly higher fractions of collagen type II and glycosaminoglycan as compares with articular chondrocytes [27]. The use of two different cell types therefore facilitated our objective of engineering cartilage constructs with differing extracellular matrix composition, and hence differing mechanical properties. Constructs were mechanical tested in either confined or unconfined compression. The choice of two testing protocols was based on the fact that (i) they represent the two most common testing modes for tissue engineered cartilage, and (ii) the type of deformation within the tissue during confined and unconfined compression is different, and one would therefore expect different correlations between the biochemical content and the equilibrium modulus in confined and unconfined compression.

In the first experiment, the equilibrium modulus in confined compression (the aggregate modulus) from engineered constructs initially seeded at different densities was found to positively correlate with both GAG and collagen II content. The aggregate modulus is determined from a confined compression test, where the sample is prevented from bulging radially; therefore both the GAG and collagen II content are good indicators of the tissues ability to resist pure compression. The aggregate modulus did not correlate with collagen I content, suggesting that collagen I may be an unreliable indicator of a tissues ability to resist pure compression. In the second experiment, the equilibrium modulus in unconfined compression (the Young's 
modulus) from engineered constructs cultured for either, 20,30, 40 or 80 days was found to positively correlate with GAG, collagen I and collagen II content. The Young's modulus is determined by unconfined compression testing, where the sample bulges radially during compression subjecting the tissue to both compressive (in the direction of loading) and tensile deformations (in the radial direction). This may explain why the collagen I content correlated with the equilibrium modulus in unconfined compression (determined from a test where the type I collagen can help support the tensile component of the deformation) in the time-in-culture experiment, and not with the equilibrium modulus in confined compression in the cell seeding density experiment. However given that collagen I was present in such small amounts in this study, it is difficult to make firm conclusions regarding its mechanical effects. The equilibrium modulus in unconfined compression of the constructs also correlated with the ratio of mature to immature collagen cross-links, which is to be expected as cross-linking is considered a good measure of construct maturity. However the equilibrium modulus in unconfined compression did not correlate with hydroxyproline, suggesting that other collagens were present, and that hydroxyproline may be an unreliable indicator of mechanical quality of tissue engineered cartilage. Although hydroxyproline content was not measured in the first experiment, given the strong correlation with collagen II, it would be expected that hydroxyproline content would have correlated with the equilibrium modulus in confined compression.

While no statistical difference was observed between the equilibrium modulus in confined compression of scaffolds seeded at different seeding densities, it would appear that an optimal seeding may exist for engineering cartilage in a semi-dynamic culture. Seeding at too high a seeding density not only reduces the amount of nutrients/growth factors per cell, but most likely also limits the diffusion of nutrients 
throughout the scaffold, resulting in an more inhomogeneous construct after static culture. Also, in time-in-culture experiment, study A, no significant difference was observed in the mechanical properties between day 40 and day 80 , despite the fact that there was an increase in the total amount of matrix synthesised (result not shown). This may also be due to the fact that the engineered construct has become more inhomogeneous in nature over time due to diffusion limitations as the tissue matures. Computational modelling has shown that as the inhomogenity of a tissue engineered cartilage construct increases, the mechanical properties of the cartilage component of the construct are increasingly underestimated by mechanical testing [28].

The time-in-culture experiment, where constructs were cultured for either 20, 30, 40 or 80 days, was replicated in two laboratories using the same study protocol but with bovine chondrocytes and sera from different sources. While significant differences were observed between the means of the mechanical properties for the constructs from the two laboratories [studies A and B (Fig. 4)], little difference was observed between the means of the biochemical content at each time point (except for the differences at day 80 reported earlier). It would therefore seem that although the amount of extracellular matrix synthesised in constructs from the two studies differs, which partially explains the difference in mechanical properties, the proportion of different matrix components (collagen I, II and GAG) present within an engineered cartilage tissue remains reasonably constant over the culture time used here. The large difference between the mean ratio of mature to immature cross-links between the two samples after 80 days suggests that the cross-linking ratio may not only depend on the time in culture, but also on the maturity of the tissue. Regardless of these findings from the means of the data, correlations between the mechanical properties and the 
biochemical content were found. The results of this study suggest that the mechanical quality of tissue engineered cartilage may be predicted with some confidence on the basis of quantification of both collagen II and GAG. Either biochemical marker on its own may not be sufficient to make firm conclusions about the mechanical quality of the tissue, particularly in unconfined compression, where weaker correlations were observed between the equilibrium modulus and either collagen II and GAG than was observed in confined compression. This may be due in part to the more complex multi-axial deformation set-up in the tissue during unconfined compression.

\section{Acknowledgements}

This work was funded by the European Union under the SCAFCART project (contract G5RD-CT-1999-00050). APH is funded in part by an endowed chair from the UK Arthritis Research Campaign. 


\section{References}

1. Mow, V.C., Gu, W.Y., Chen, F.H., Structure and function of articular cartilage and meniscus. In: Basic orthopedic biomechanics and mechano-biology, Mow, V.C., Huiskes, R. (Eds), $3^{\text {rd }}$ edition, Lippincott Williams and Wilkins, Philadelphia, Chapter 5, 2005.

2. Maroudas, A. Physicochemical properties of articular cartilage. In: Freeman, M.A.R. (Ed.). Adult Articular Cartilage, $2^{\text {nd }}$ ed., Pitman Medical, Kent, England, 215-290, 1979.

3. Soltz, M.A., Ateshian, G.A. Experimental verification and theoretical prediction of cartilage interstitial fluid pressurization at an impermeable contact interface in confined compression. J. Biomech., 31, 927-934, 1998.

4. Hunter, C.J., Mouw, J.K., Levenston, M.E. Dynamic compression of chondrocyte-seeded fibrin gels: effects on matrix accumulation and mechanical stiffness. Osteoarthr. Cartil., 12, 117-130, 2003.

5. Mauck, R. L., Seyhan, S. L., Ateshian, G. A., Hung, C. T. Influence of seeding density and dynamic deformational loading on the developing structure/function relationships of chondrocyte-seeded agarose hydrogels. Ann. Biomed. Eng., 30, 1046-1056, 2002.

6. Vunjak-Novakovic, G., Martin, I., Obradovic, B., Treppo, S., Grodzinsky, A.J., Langer, R., Freed, L.E. Bioreactor cultivation conditions modulate the composition and mechanical properties of tissue-engineered cartilage. J. Orthop. Res., 17: 130-138, 1999.

7. Buschmann, M.D., Gluzband, Y.A., Grodzinsky, A.J., Kimura, J.H., Hunziker, E.B. Chondrocytes in culture synthesize a mechanically functional extracellular matrix. J. Orthop. Res., 10: 745-758, 1992. 
8. Rotter, N., Tobias, G., Lebl, M., Roy, A. K., Hansen, M. C., Vacanti, C. A, Bonassar, L. J. Age-related changes in the composition and mechanical properties of human nasal cartilage. Arch. Biochem. Biophys., 403, 132-140, 2002.

9. Gooch, K. J., Blunk, T., Courter, D. L., Sieminski, A. L., Bursac, P.M., Vunjak-Novakovic, G., and Freed, L. E. IGF-I and mechanical environment interact to modulate engineered cartilage development. Biochem. Biophys. Res. Commun., 286, 909-915, 2001.

10. Fedewa M.M., Oegema T.R. Jr., Schwartz M.H., MacLeod A., Lewis J.L. Chondrocytes in culture produce a mechanically functional tissue J. Orthop. Res., 16, 227-236, 1998.

11. Mauck, R.L., Nicoll, S.B., Seyhan, S.L, Ateshian, G.A., Hung, C.T. Synergistic action of growth factors and dynamic loading for articular cartilage tissue engineering. Tissue Eng., 9, 597-611, 2003.

12. Stading M., Langer R. Mechanical shear properties of cell-polymer cartilage constructs. Tissue Eng., 5, 241-250, 1999.

13. Carver, S.E., Heath, C.A. Semi-continuous perfusion system for delivering intermittent physiological pressure to regenerating cartilage. Tissue Eng., 5, 1$11,1999$.

14. Freed, L.E., Vunjak-Novakovic, G., Biron, R.J., Eagles, D.B., Lesnoy, D.C., Barlow, S.K., Langer, R. Biodegradable polymer scaffolds for tissue engineering. Biotechnology, 12, 689-693, 1994.

15. Martin I., Obradovic B., Treppo S., Grodzinsky A.J., Langer R., Freed L.E., Vunjak-Novakovic G. Modulation of the mechanical properties of tissue engineered cartilage. Biorheology, 37, 141-147, 2000. 
16. Pei, M., Seidel, J., Vunjak-Novakovic, G., Freed, L.E. Growth factors for sequential cellular de- and re-differentiation in tissue engineering. Biochem. Biophys. Res. Commun.,. 294, 149-154, 2002.

17. Williams, G.M., Klein, T.J., Sah, R.J. Cell density alters matrix accumulation in two distinct fractions and the mechanical integrity of alginate-chondrocyte constructs Acta Biomater., 1, 625-633, 2005.

18. Ng, K.W., Wang, C.C.-B., Mauck, R.L., Kelly, T.-A.N., Chahine, N.O., Costa, K.D.; Ateshian, G.A.; Hung, C.T.A layered agarose approach to fabricate depth-dependent inhomogeneity in chondrocyte-seeded constructs. J. Orthop. Res., 23, 134-141, 2005.

19. Waldman, S.D., Couto, D.C., Grynpas, M.D., Pilliar, R.M., Kandel, R.A. A single application of cyclic loading can accelerate matrix deposition and enhance the properties of tissue-engineered cartilage. Osteoarthr. Cartil., 14, 323-330, 2006.

20. Dickinson S.C., Sims T.J., Pittarello L., Soranzo C., Pavesio A. \& Hollander A.P. Quantitative outcome measures of cartilage repair in patients treated by tissue engineering. Tissue Eng., 11, 277-87, 2005.

21. Crawford, A. and Dickinson, S.C. Chondrocyte isolation, expansion and culture on polymer scaffolds. In: Biopolymer Methods in Tissue Engineering, p147. Editors A.P. Hollander and P.V. Hatton. Humana Press. 2004.

22. Iwata Y., Bourne R., Rorabeck C., Poole A.R. Increased damage to type II collagen in osteoarthritic articular cartilage detected by a new immunoassay. J. Clin. Invest., 93, 1722-32, 1994.

23. Kafienah W., Sims T.J. Biochemical methods for the analysis of tissue engineered cartilage. Methods Mol. Biol., 238, 217-30, 2004. 
24. Handley C.J., Buttle D.J. Assay of proteoglycan degradation. Methods Enzymol., 248, 47-58, 1995.

25. Puelacher, W.C., Mooney, D., Langer, R., Upton, J., Vacanti, J.P., and Vacanti, C.A. Design of nasoseptal cartilage replacements synthesized from biodegradable polymers and chondrocytes. Biomaterials 15, 774, 1994.

26. Naumann, A., Rotter, N., Bujia, J., and Aigner, J. Tissue engineering of autologous cartilage transplants for rhinology. Am. J. Rhinol. 12, 59, 1998.

27. Kafienah, W., Jakob, M., Démarteau, O., Frazer, A., Barker, M.D., Martin, I., Hollander, A.P. Three-dimensional tissue engineering of hyaline cartilage: Comparison of adult nasal and articular chondrocytes. Tissue Eng., 8, 817826, 2002.

28. Kelly, D.J., Prendergast, P.J. Effect of a degraded core on the mechanical behaviour of tissue-engineered cartilage constructs: a poro-elastic finite element analysis. Med. Biol. Eng. Comput., 42, 9, 2004. 


\section{List of figures}

Fig. 1. (a) Representative force versus time curve for a stress relaxation test. A ramp displacement of $10 \%$ strain was applied to each sample, which was then held until the measured reaction force equilibrated. Two subsequent ramp displacements of $5 \%$ strain each were then applied to each sample, giving a total strain of $20 \%$. (b) Based on the magnitude of the equilibrium force measured after each ramp and hold, a stress-strain curve for the tissue can be obtained. The aggregate or Young's modulus of the tissue is obtained from the slope of the stress-strain curve.

Fig. 2. Influence of cell seeding density on the (a) aggregate modulus, (b) collagen I, (c) collagen II and (d) GAG content (expressed as a percentage of construct dry weight) of tissue engineered cartilage. Plots represent mean \pm SEM. (* Significant difference between groups, $\mathrm{p}<0.05)$.

Fig. 3. Correlations between aggregate modulus and (a) collagen I, (b) collagen II and (c) GAG content for the cell seeding density experiment. $r \sim$ Spearman correlation coefficient.

Fig. 4. Influence of culture time on the Young's modulus, collagen I, II, GAG, hydroxyproline content (expressed as a percentage of construct dry weight) and the ratio of mature to immature cross-links of tissue engineered cartilage. Data is shown 
for Study A and Study B. Numbers above bars indicate level of difference between Study A and Study B. Lines indicate difference due to time-in-culture $(* \sim p<0.05$; $* * \sim \mathrm{p}<0.01 ; * * * \sim \mathrm{p}<0.001)$. Lines above bars represent differences in original experiment, lines below differences in replicate. (! For original experiment, significant difference observed between all time points with $\mathrm{p}<0.001$, except between day $30 \&$ day 40 , where $\mathrm{p}<0.01)$.

Fig. 5. Correlations between aggregate modulus and (a) collagen $I$, (b) ratio of immature: mature collagen cross-links, (c) collagen II, (d) hydroxyproline and (e) GAG content for the time-in-culture experiment. $r \sim$ Spearman correlation coefficient. 
Figures

(a)

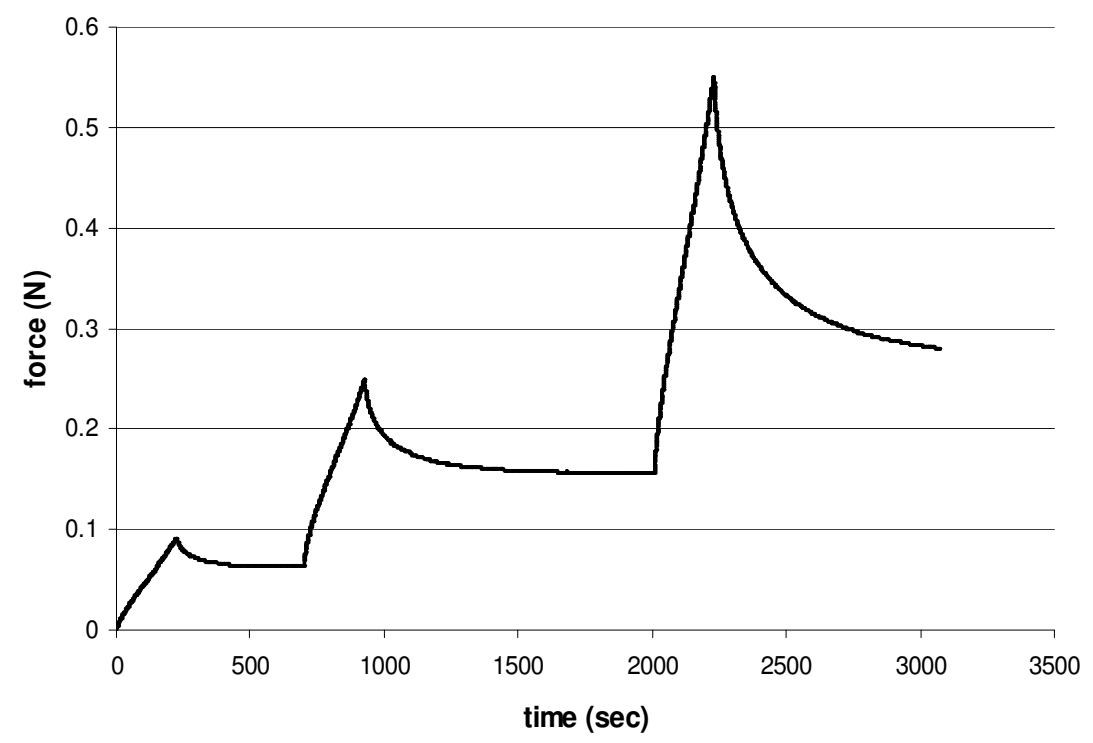

(b)

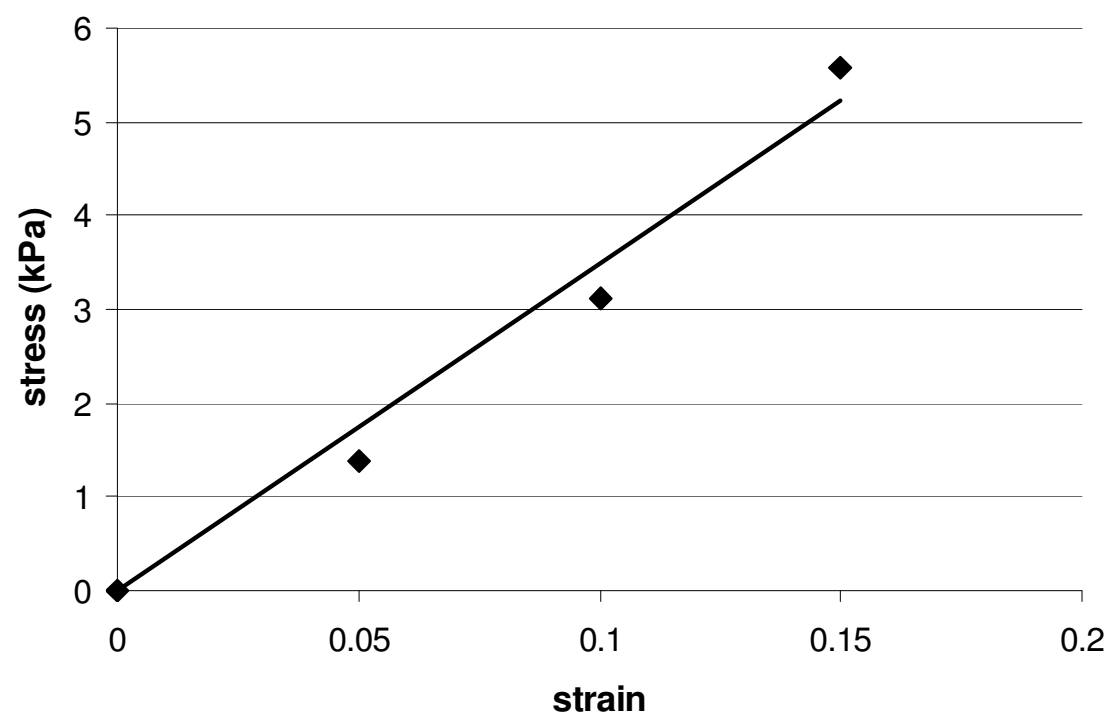

Fig. 1. 


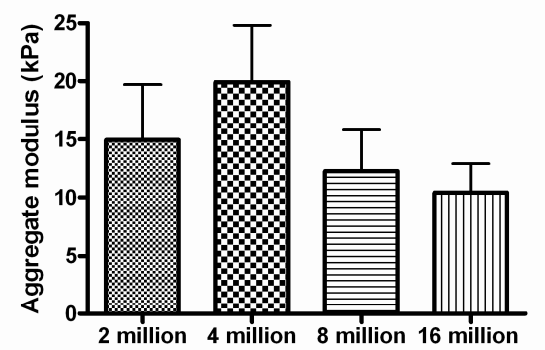

(a) Seeding density (cells/scaffold)

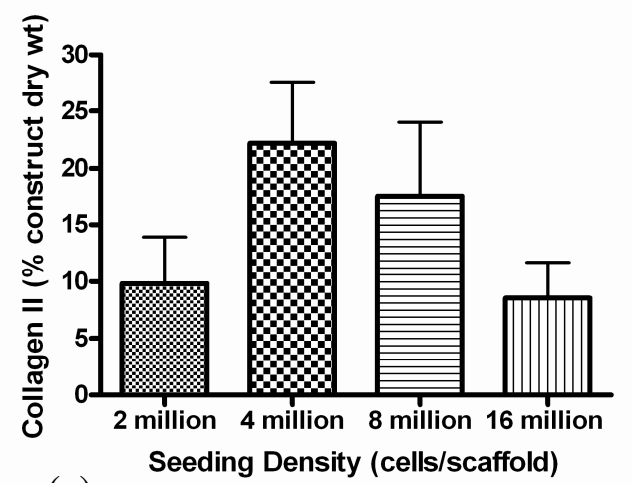

(c)

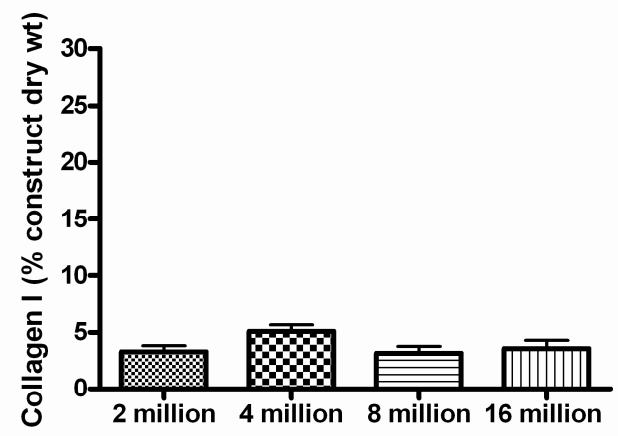

(b) Seeding Density (cells/scaffold)

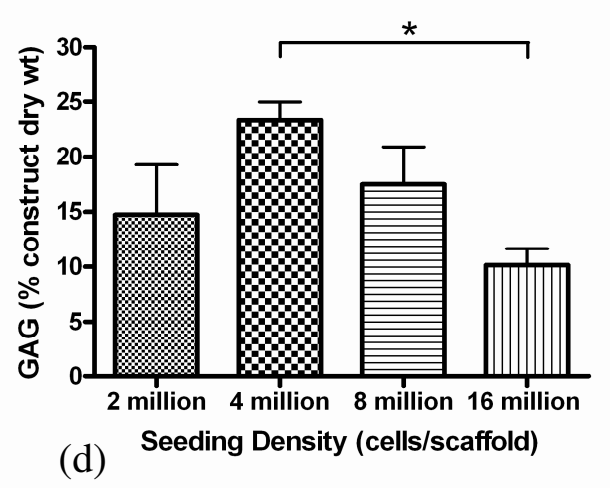

Fig. 2. 
(a)

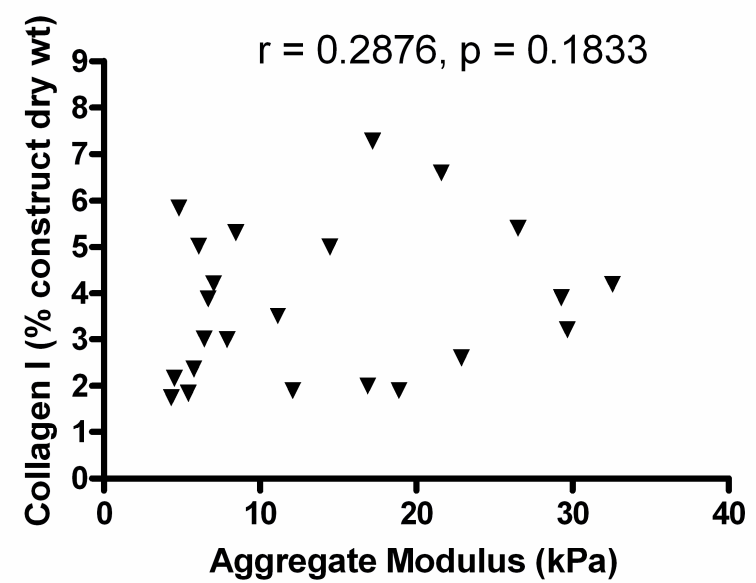

(b)

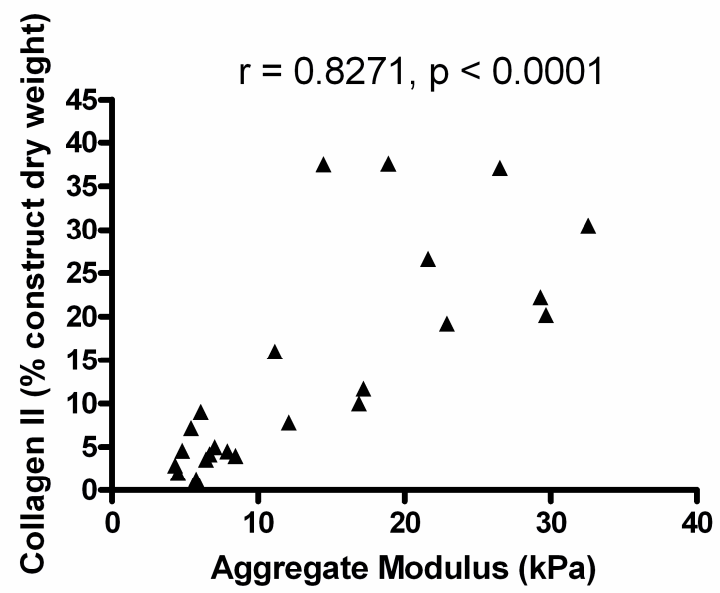

(c)

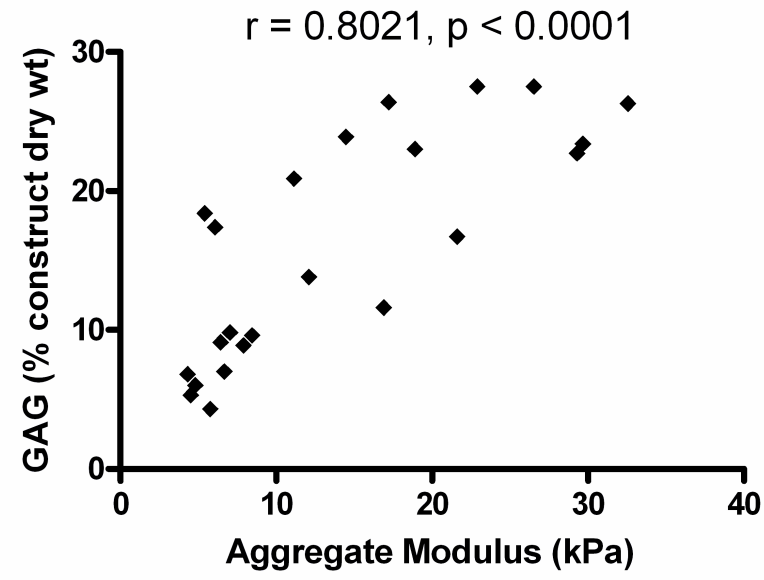

Fig. 3. 


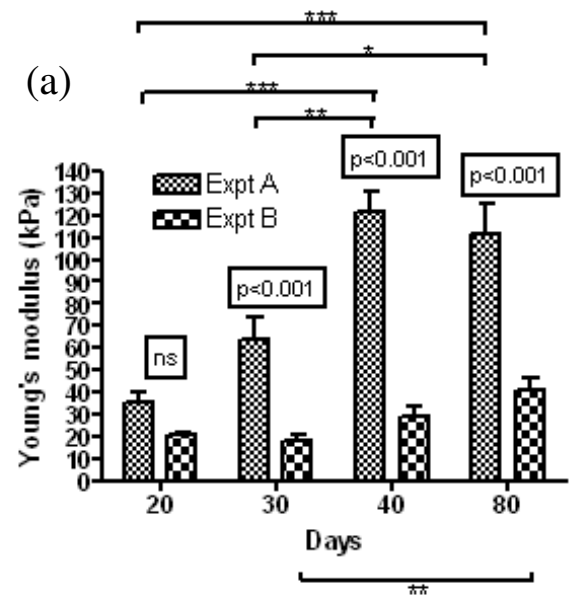

(b)

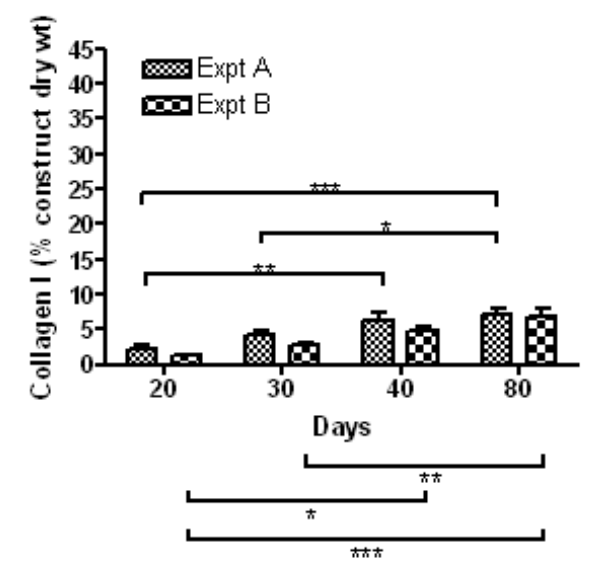

(c)

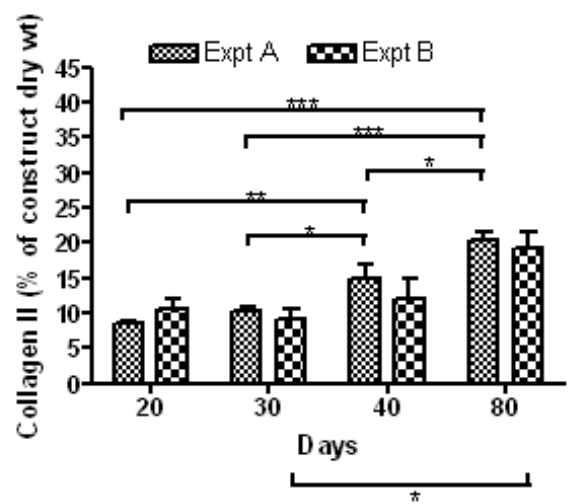

(d) $\quad \quad \quad m E x p t A \quad \infty \quad \infty x p t B \quad p<0.05$

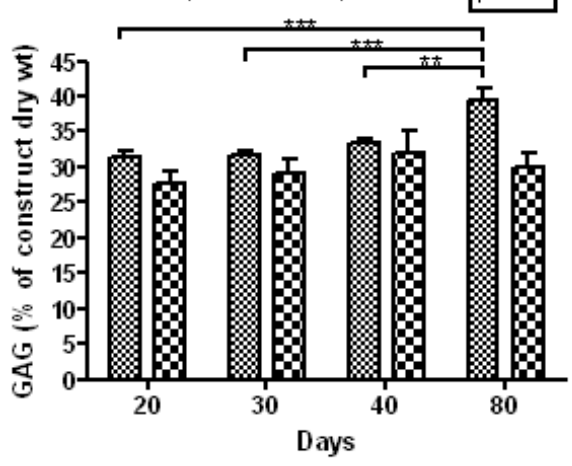

(e)

(f)
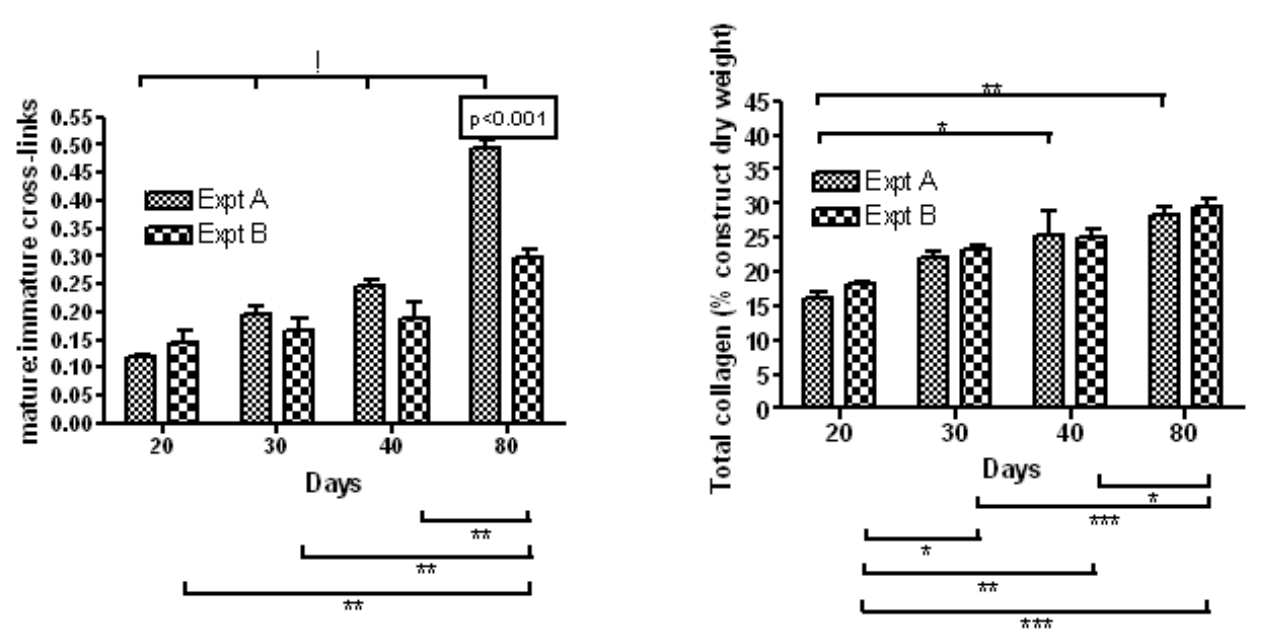

Fig. 4. 

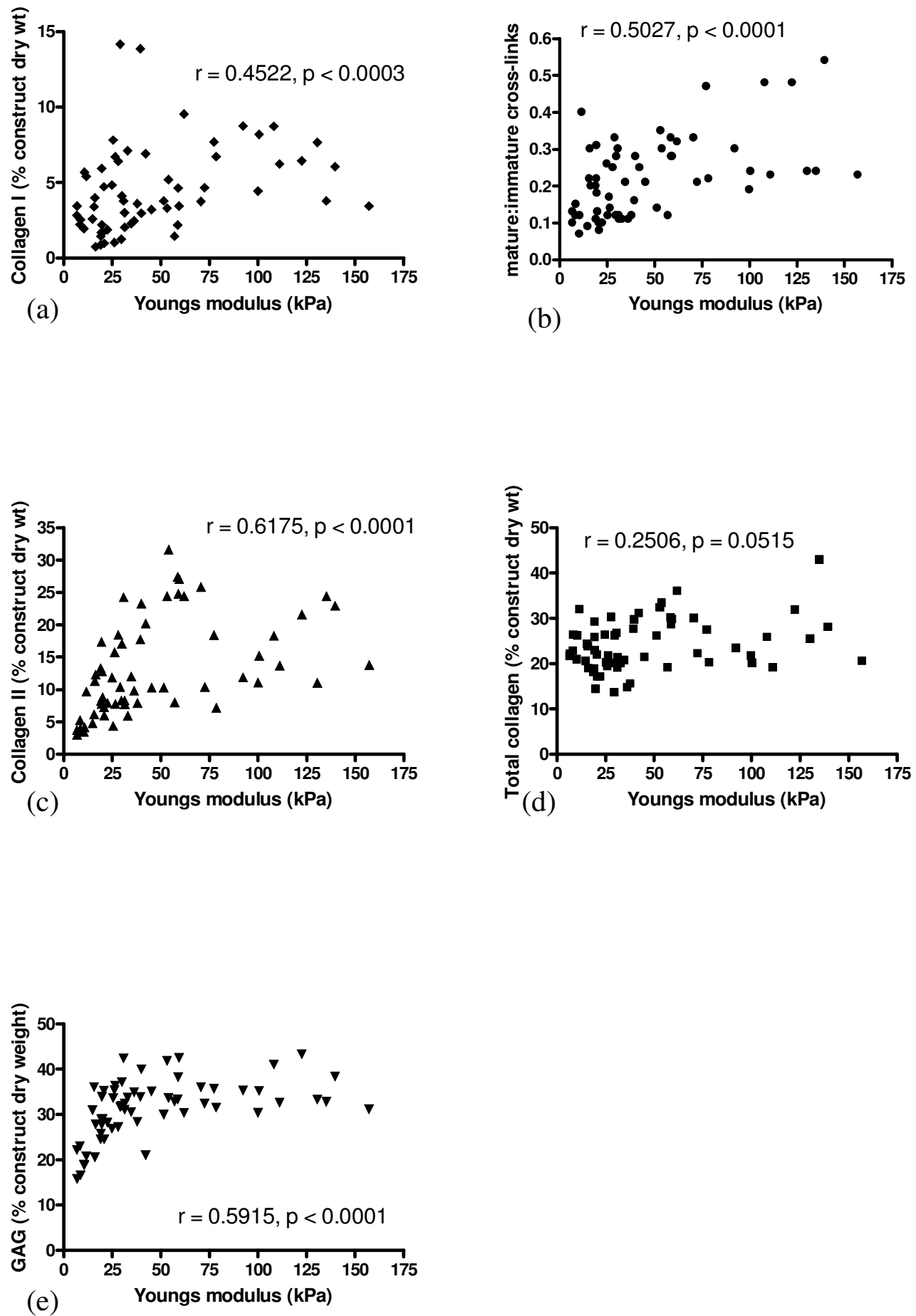

Fig. 5. 